\title{
Erratum: Unwinding of strings thrown into a fuzzball
}

\author{
Stefano Giusto ${ }^{a, b}$ and Samir D. Mathur ${ }^{c}$ \\ ${ }^{a}$ Dipartimento di Fisica, Università di Genova, \\ via Dodecaneso, 33, 16146 Genova, Italy \\ ${ }^{b}$ INFN, Sezione di Genova, \\ via Dodecaneso, 33, 16146 Genova, Italy \\ ${ }^{c}$ Department of Physics, The Ohio State University, \\ Columbus, OH 43210, U.S.A. \\ E-mail: stefano.giusto@cea.fr, mathur@mps.ohio-state.edu
}

ERratum to: JHEP07(2010)009

The correct address of Stefano Giusto is:

Dipartimento di Fisica, Università di Genova, via Dodecaneso, 33, 16146 Genova, Italy and

INFN, Sezione di Genova, via Dodecaneso, 33, 16146 Genova, Italy 